\title{
Which methods for bedside Bayes?
}

Understanding how and why Bayes theorem translates pre-test into post-test probabilities is the pons asinorum (Euclid's 5th proposition is known as the "bridge of asses" because many folk got stuck crossing it) of evidence-based medicine (EBM). Because different minds are sparked by different methods, we teach the theorem by using several presentations: $2 \times 2$ tables, trees, geometric figures, and formulas. A good teacher will have several of these in his or her armamentarium. ${ }^{1}$ However, having crossed the bridge of understanding, we still must cross the bridge to practice. The basic understanding is essential, but the time pressures of clinical practice require that we abandon the training wheels and move to tools of rapid calculation. No single tool will suit all people and circumstances, so a well-stocked tool box is advisable. In this editorial, I describe 3 methods aimed primarily at everyday practice rather than enhancing understanding: pre-calculated tables or graphs, programmed Bayes calculators, and the Bayes nomogram.

\section{Pre-calculated tables and graphs}

Applying Bayes theorem in clinical practice will be quicker if the calculations are already done. For specific common tests, this pre-calculation can be achieved by either tabulating or graphing the post-test probabilities for all plausible pre-test probability values. $^{2}$ The calculation becomes a simple look-up (provided you have properly organised your information about the test). Ideally, software for critically appraised topics should include the ability to produce such graphs or tables. Figure 1 shows a graph version of conjunctival pallor for the diagnosis of anaemia."

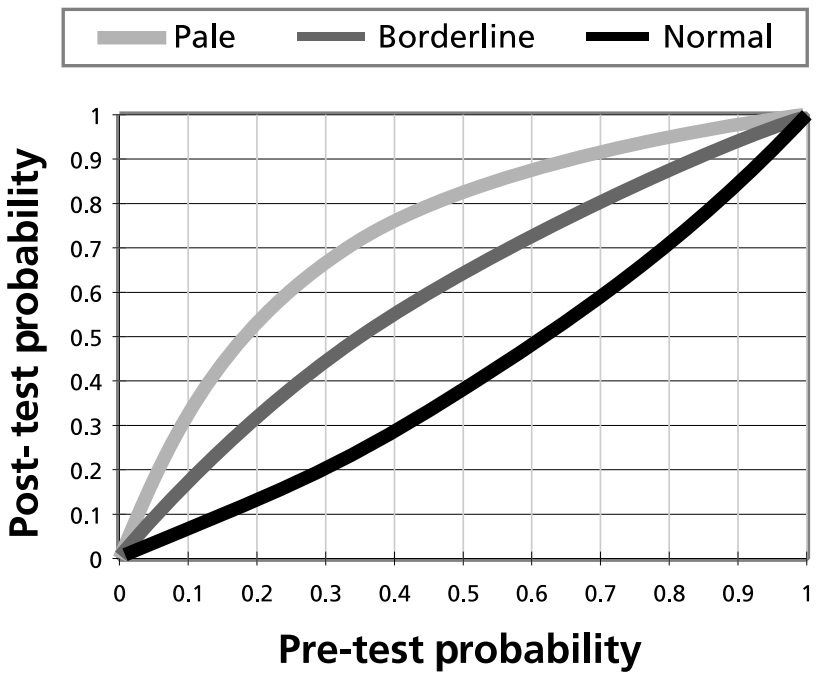

Figure 1 Pre-and post-test probabilities of anaemia for 3 categories of conjunctival appearance: pale (little or no red colour), borderline (neither clearly red nor clearly pale), and normal. ${ }^{3}$
The method is possible for both dichotomous and multicategory tests. Spreadsheets that do such calculations are readily available. ${ }^{4}$ The results can be kept on a computer or a handheld or printed on paper. Another advantage is that common pre-test probabilities can be marked and kept directly on the graph, acting as a guide and anchor for the individual patient being assessed. Given their simplicity, we recommend having such graphs or tables available for your most frequently used tests. However, you will still need another method when applying a test not in your current collection.

\section{Programmed Bayes calculators}

The presence of computers or handhelds at the point of care has simplified doing exact Bayes calculations. Many programs are available: spreadsheets ${ }^{4}$ that require you to have the appropriate spreadsheet software, internet versions that require you only to have a Java-enabled web browser and an internet connection, ${ }^{45}$ and purpose-built Bayes calculators for personal computers and handhelds. ${ }^{4}$ Figure 2 shows an Excel spreadsheet that allows entry of the 3 required probabilities, then calculates the post-test probabilities and also provides a graphic presentation of the results.

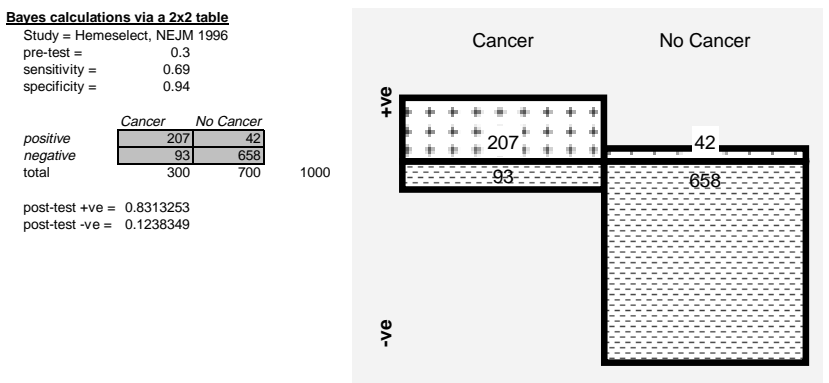

Figure 2 An Excel spreadsheet for calculating post-test probabilities for dichotomous tests.

Such programs simply require the entry of the necessary test characteristics and pre-test probability. This method is faster than a calculator, but the requirement to look up the test characteristics and enter the numbers is still more tedious than the pre-calculation described above. However, every self-respecting EBMer should carry one such program (if for no other purpose than being able to confidently check the calculations of students).

\section{Bayes nomogram}

For the price of a laminated strip of paper, you can obtain the simplest of the Bayes calculators-the Bayes nomogram. First described by Fagan $^{6}$ in the days before personal computers and 


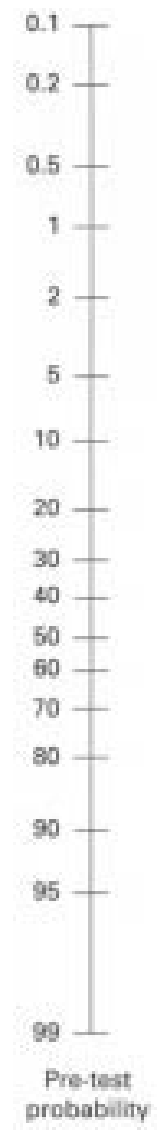

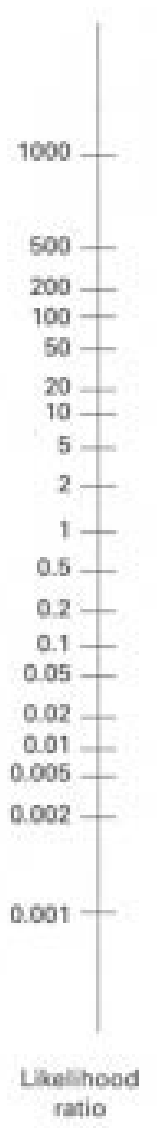

Figure 3 Bayes nomogram (adapted from Fagan). ${ }^{6}$

handhelds, the nomogram has had an enduring popularity. A typical nomogram is shown in Figure 3. The only requirements for calculating the post-test probability are the pre-test probability, the relevant likelihood ratio (LR), and an implement with a straight edge (although a non-arthritic finger can substitute).

The construction of the nomogram relies on being able to convert Bayes theorem into a simple linear additive function. A couple of mathematical tricks can achieve this. First, we need the odds version of Bayes theorem:

Post-test odds $=$ Pre-test odds $\times$ LR

or in mathematical notation:

$$
\mathrm{O}(\mathrm{D} \mid \mathrm{R})=\mathrm{O}(\mathrm{D}) \times \mathrm{P}(\mathrm{R} \mid \mathrm{D}) / \mathrm{P}(\mathrm{R} \mid \mathrm{NonD})
$$

where $\mathrm{O}(\mathrm{D})$ is the odds of the disease, which is $\mathrm{O}(\mathrm{D})=$ $\mathrm{P}(\mathrm{D}) /(1-\mathrm{P}([\mathrm{D}])$, and $\mathrm{R}$ is a specific test result. Taking logarithms converts this to a simple addition, which allows the ruler to do the work:

$$
\log (\text { Post-test odds })=\log (\text { Pre-test odds })+\log (\mathrm{LR})
$$

or in mathematical notation:

$$
\log (\mathrm{O}[\mathrm{D} \mid \mathrm{R}])=\log (\mathrm{O}[\mathrm{D}])+\log (\mathrm{P}[\mathrm{R} \mid \mathrm{D}] / \mathrm{P}[\mathrm{R} \mid \mathrm{NonD}]) .
$$

Although instructive for students, the nomogram has several limitations. First, it requires knowing the LRs, which often means looking up the sensitivity and specificity and then converting these to LRs. For example, with a dichotomous test, the positive LR is sensitivity/(1-specificity), and the negative LR is (1-sensitivity)/specificity. Second, the nomogram has limited accuracy, although it is generally sufficient for bedside calculations. Finally, the pre- and post-test ranges of the nomogram are limited (generally from 0.001 to 0.990 ), which may be inadequate for some screening test problems.

\section{Conclusions}

The 3 methods described in this editorial are for bedside calculation. For teaching, other methods, such as $2 \times 2$ tables or box diagrams, are more helpful for gaining insight into the need for and mechanism of Bayes theorem. Having gained that insight, students should then be able to rapidly experiment with calculations to learn the consequences of changing test characteristics and pre-test probabilities. With the advent of handheld computers, we believe that pre-calculation and software will largely take over from the nomogram; hence, we have dropped it from our glossary.* Readers are invited to use the methods described above ${ }^{4}$ and send us comments on how the presentation of test probabilities may be made more useful at the bedside.

PAUL GLASZIOU, MBBS, PHD University of Queensland Herston, Queensland, Australia

1 Sackett DL, Straus SE, Richardson WS, Rosenberg W, Haynes RB. Evidence-based medicine: how to practice and teach EBM. Second edition. Edinburgh: Churchill Livingstone, 2000.

2 Glasziou PP. Probability revision. Prim Care 1995;22:235-45.

3 Sheth TN, Choudhry NK, Bowes M, Detsky AS. The relation of conjunctival pallor to the presence of anaemia. J Gen Intern Med 1997;12:102-6.

4 http://www.cebm.utoronto.ca/practise/ca/statscal/

5 http://www.health.usyd.edu.au/ebm/bayes.htm and http://www.intmed. mcw.edu/clincalc/bayes.html can be used online or offline (click on "Make available offline").

6 Fagan TJ. Nomogram for Bayes theorem [Letter]. $N$ Engl J Med 1975;293:275.

*The Bayes nomogram that appeared in the glossary of Evidence-Based Medicine in the July/August, September/October and November/December 1999 issues should not be used because the likelihood ratio is imperfectly drawn, giving inaccurate readings in parts of the nomogram; and the lower 500 on the likelihood ratio scale should be 200. 


\section{Journals reviewed for this issue*}

Acta Obstet Gynecol Scand
Age Ageing
Am J Cardiol
Am J Med
Am J Obstet Gynecol
Am J Psychiatry
Am J Public Health
Am J Respir Crit Care Med
Ann Emerg Med
Ann Intern Med
Ann Surg
Arch Dis Child
Arch Gen Psychiatry
Arch Intern Med
Arch Neurol

Arch Pediatr Adolesc Med
Arch Surg
Arthritis Rheum
BJOG
BMJ
BrJ Gen Pract
BrJ Psychiatry
BrJ Surg
CMAJ
Chest
Circulation
Cochrane Library
Crit Care Med
Diabetes Care
Gastroenterology

Gut

Heart

Hypertension

JAMA

J Am Coll Cardiol

J Am Coll Surg

J Am Geriatr Soc

J Clin Epidemiol

J Fam Pract

J Gen Intern Med

J Infect Dis

J Intern Med

J Neurol Neurosurg Psychiatry

$\mathrm{J}$ Pediatr
J Vasc Surg

Lancet

Med Care

Med J Aust

N Engl J Med

Neurology

Obstet Gynecol

Pain

Pediatrics

Rheumatology

Spine

Stroke

Surgery

*Approximately 60 additional journals are reviewed. This list is available on request.

How to cite material from Evidence-Based Medicine

Citation of material from the Notebook

Milne R, Hicks N. Evidence-based purchasing [EBM Note]. Evidence-Based Medicine 1996 May-Jun;1:101-2.

Citation for material taken from a structured abstract, written without attribution by a staff member

Antihypertensive drugs decrease mortality, coronary events, and stroke in elderly persons [abstract]. Evidence-Based Medicine 1996 May-Jun;4:105. Abstract of: Pearce KA, Furberg CD, Rushing J. Does antihypertensive treatment of the elderly prevent cardiovascular events or prolong life? A meta-analysis of hypertension treatment trials. Arch Fam Med 1995;4:943-50.

Citation for material taken from a commentary to an article

Olds D. Commentary on "Home visiting programmes reduce childhood injury." Evidence-Based Medicine 1996 May-Jun;4:112. Comment on: Roberts I, Kramer MS, Suissa S. Does home visiting prevent childhood injury? A systematic review of randomised controlled trials. BMJ 1996;312:29-33. 